possédons sont tout à fait insuffisantes; en conséquence il propose la résolution no. 2 , qui est approuvée par la Commission.

Le "Draft Report" est adopté avec une modification proposée par Mlle Harwood.

\title{
Résolutions adoptées
}

I. La Commission recommande aux observateurs de Jupiter et de Saturne au télescope, de porter plus systématiquement leur attention sur l'étude des couleurs, des niveaux et des mouvements des taches de ces planètes.

2. La Commission exprime le vœu que les physiciens expérimentaux possédant l'équipement nécessaire entreprennent l'étude de l'équation d'état des gaz simples, en particulier l'hydrogène, l'hélium, le méthane et l'ammoniac jusqu'aux pressions les plus hautes que l'on puisse atteindre.

\section{COMMISSION I8 (LONGITUDES)}

\section{(COMMISSION INTERNATIONALE MIXTE DES LONGITUDES)}

\author{
President: Général G. Perrier. \\ Secretary: Dr A. LAMberT.
}

La Commission internationale mixte des Longitudes a tenu deux séances: la première le vendredi 5 août, à $9^{\mathrm{h}} 30^{\mathrm{m}}$, la deuxième commune avec l'une des séances de la Commission de l'Heure, le samedi 6 août, à $10^{\mathrm{h}} 30^{\mathrm{m}}$.

Ière séance. Vendredi, 5 août 1938.

M. A. Lambert donne lecture du Rapport présenté à la Commission.* L'impression du Mémoire d'ensemble, relatif aux travaux de l'Opération internationale des Longitudes de I933, est commencée. Soixante et onze stations ont transmis leurs observations au Bureau international de l'Heure qui les a discutées. La propagation des ondes a fait également l'objet d'une étude dont on apporte les conclusions.

L'introduction contenant l'historique et l'exposé des méthodes de réductions employées, d'une part, et la monographie relative à la station d'Adelaïde de l'autre, constituent les deux premiers fascicules imprimés de la publication, soumis pour examen à la Commission.

L'ordre du jour des travaux de la Commission est ainsi fixé:

I. Influence du mouvement du pôle sur la longitude d'un point. (Proposition de M. F. da Costa Lobo.)

2. Quelle est la meilleure manière de traiter la question de l'équation personnelle dans les observations de passage? (Proposition de M. H. M. Jeffers.)

3. Communication de MM. R. Jouaust et N. Stoyko sur Les phénomènes de propagation des ondes radioélectriques et leur influence sur les opérations de détermination de longitude.

4. Détermination précise de la longitude par observations de distances zénithales extra-méridiennes au théodolite, par la méthode de l'obturateur oculaire. (Proposition de M. J. de Graaff Hunter.)

$$
\text { * P. I2I. }
$$


5. Publication du Mémoire relatif à l'Opération internationale des Longitudes de 1933. (Devis, couverture des dépenses, volume unique ou fascicules séparés.)

6. L'Opération des Longitudes devra-t-elle être répétée et quand? (Proposition de M. H. M. Jeffers.)

Les questions $I, 2$ et 4 sont abordées à cette première séance.

Question I. M. A. Lambert fait connaître que l'on a tenu compte du mouvement du pôle dans les calculs relatifs à l'Opération des Longitudes de I933 et que l'influence de ce mouvement est également prise en considération dans les données définitives publiées annuellement par le Bureau international de l'Heure.

Question 2. L'influence de l'équation personnelle dans les observations de passage et la qualité des appareils propres à la mesurer donne lieu à une discussion à laquelle prennent part MM. H. M. Jeffers, A. Lambert, H. Spencer Jones, N. Stoyko, N. E. Nörlund, V. V. Michkovitch, F. Moreau, G. Silva, J. de Graaff Hunter, M. Reda Madwar, K. D. P. Rosén, S. Plakidis. L'ordre de grandeur de cette équation, usage étant fait du micromètre dit impersonnel, est très diversement estimé par des astronomes.

Question 4. M. J. de Graaff Hunter présente la maquette d'un dispositif oculaire qu'il a imaginé. Il fait connaître la méthode d'observation à employer. La suite de son exposé est renvoyée à la séance suivante.

zème séance. Samedi, 5 aout 1938 .

Cette séance est commune aux Commissions des Longitudes (I8) et de l'Heure (3I). Le Bureau est constitué par les Présidents des deux Commissions, le Général G. Perrier et M. N. E. Nörlund, assistés de M. A. Lambert, Secrétaire.

Les questions $4,3,5$ et 6 figurant à l'ordre du jour de la Commission sont examinées au cours de cette séance.

Question 4. M. J. de Graaff Hunter poursuit l'exposé qu'il a commencé à la séance de la veille, sur la détermination précise des longitudes au moyen de l'obturateur oculaire.*

Cet accessoire confère au théodolite les avantages du micromètre impersonnel de l'instrument méridien.

M. de Graaff Hunter fait connaître les résultats de mesures effectuées à Cottenham en novembre I937 grâce au dispositif qu'il a imaginé. En moins de deux heures et par l'observation de six paires d'étoiles, on obtient, aux latitudes moyennes, la longitude avec $\pm 0.04 \mathrm{sec}$. d'erreur moyenne en faisant usage d'un petit théodolite de ro pouces de distance focale.

Question 3. M. N. Stoyko donne lecture d'une étude sur les Phénomènes de propagation des ondes radioélectriques et leur influence sur les opérations de détermination des longitudes, par R. Jouaust et N. Stoyko. $\dagger$

Il s'agit des précautions à prendre pour l'utilisation des ondes courtes dans les mesures précises. Leur mode de propagation dans la haute atmosphère, variable le jour, la nuit, et suivant les saisons, conduit à des vitesses apparentes différentes. La durée exacte de propagation ne peut être calculée que si l'on connaît l'angle d'incidence. Les radiotélégraphistes savent aujourd'hui mesurer cet angle, au moyen d'un matériel, important il est vrai et d'emploi délicat.

Question 5. Le Président présente à la Commission les deux premiers fascicules

* Voir Proceedings of the Royal Society (Series A, no. 955, Vol. 166, pp. 197-213, May 1938) et Bulletin géodésique (1938, p. I89).

$\dagger$ Bulletin géodtsique, r938, p. 23. 
parus* du Mémoire d'ensemble sur l'Opération des Longitudes de I933, ainsi que le devis des dépenses totales qu'il faut envisager pour l'impression.

Question 6. M. E. Esclangon, qui estime que le problème de la variation des longitudes a pris un aspect nouveau depuis qu'il est suivi d'une façon continue par le Bureau international de l'Heure, croit qu'il n'y a pas lieu de décider actuellement la reprise d'une nouvelle Opération internationale.

La Commission, comme conclusion, adopte la résolution suivante à l'unanimité:

"La Commission internationale mixte des Longitudes a examiné les deux premiers fascicules parus de l'exposé général de l'Opération internationale des Longitudes de I933.

"Elle est reconnaissante au Gouvernement français d'avoir mis à la disposition du Bureau international de l'Heure les fonds nécessaires aux calculs et à la publication de ces deux premiers fascicules.

"La Commission recommande la continuation de la publication sous la même forme pour les soixante et onze stations de longitude qui ont participé à l'Opération, et elle demande, après avoir examiné le devis des dépenses d'impression qui lui a été soumis, qu'une subvention annuelle de $375^{\circ}$ francs or soit mise à la disposition du Président de la Commission mixte durant trois années consécutives, par chacune des Unions astronomique et géodésique-géographique. $\dagger$

"Cette publication sera la conclusion de l'Opération de r933 donnant la position en longitude de soixante et onze stations à la surface du globe.

“ $D$ 'autre part, aucune variation en longitude n'ayant été mise en évidence par la comparaison des résultats de I933 avec ceux de I926, la Commission estime qu'il n'y a pas lieu de recommencer semblable Opération avant un laps de temps de dix ou quinze années. Les deux Unions en décideront dans une de leurs Assemblées générales ultérieures."

Le Président fait connaître qu'il vient de recevoir par une lettre de M. Y. Hagihara des propositions de M. M. Miyadi.

En l'absence de MM. Y. Hagihara et M. Miyadi, acte est seulement pris de ces propositions, dont copie sera adressée pour avis à tous les membres de la Commission.

\title{
COMMISSION I9 (VARIATION OF LATITUDE)
}

(COMMISSION MIXTE DES LATITUDES)

\author{
President: Dr H. Spencer Jones. \\ SECRETARY: Mr W. D. LAMBERT.
}

The committee met at II.30 a.m. on Thursday, August 4, I938.

Prof. Carnera stated that the Draft Report had been made available to all and that he would therefore not summarize it; furthermore the results for 1936 had been published in Astronomische Nachrichten No. 6290 and those for I937 in Astrono-

\footnotetext{
* Ils ont été distribués à tous les membres de la Commission mixte des Longitudes, présents à l'Assemblée générale de Stockholm, et expédiés ensuite aux membres absents.

$\dagger$ Après réception de cette résolution, sur avis de la Commission des Finances de l'Union Astronomique Internationale et sur proposition de son Comité Exécutif, l'Assemblée Générale de l'Union a décidé d'accorder pour sa part une subvention totale de 7500 francs or, 2500 francs or par an pendant 3 ans, soit les $2 / 3$ de la demande de la Commission.
} 